\title{
Retinal vasculature enhancement using independent component analysis
}

\author{
Ahmad Fadzil M. Hani ${ }^{1 *}$, Hanung Adi Nugroho ${ }^{1,2 * *}$ \\ ${ }^{1}$ Department of Electrical and Electronic Engineering, Universiti Teknologi PETRONAS, Bandar Seri Iskandar, Tronoh, Perak Darul \\ Ridzuan, Malaysia; ${ }^{2}$ Department of Electrical Engineering, Univeristas Gadjah Mada, Jl. Grafika 2, Kampus UGM, Jogjakarta, Indo- \\ nesia. \\ Email: *fadzmo@petronas.com.my; **hanungadin@gmail.com
}

Received 22 June 2009; revised 16 July 2009; accepted 24 July 2009.

\section{ABSTRACT}

Retinal vasculature is a network of vessels in the retinal layer. In ophthalmology, information of retinal vasculature in analyzing fundus images is important for early detection of diseases related to the retina, e.g. diabetic retinopathy. However, in fundus images the contrast between retinal vasculature and the background is very low. As a result, analyzing or visualizing tiny retinal vasculature is difficult. Therefore, enhancement of retinal vasculature in digital fundus image is important to provide better visualization of retinal blood vessels as well as to increase accuracy of retinal vasculature segmentation. Fluorescein angiogram overcomes this imaging problem but it is an invasive procedure that leads to other physiological problems. In this research work, the low contrast problem of retinal fundus images obtained from fundus camera is addressed. We develop a fundus image model based on probability distribution function of melanin, haemoglobin and macular pigment to represent melanin, retinal vasculature and macular region, respectively. We determine retinal pigments makeup, namely macular pigment, melanin and haemoglobin using independent component analysis. Independent component image due to haemoglobin obtained is used since it exhibits higher contrast retinal vasculature. Contrast of retinal vasculature from independent component image due to haemoglobin is compared to those from other enhancement methods. Results show that this approach outperforms other non-invasive enhancement methods, such as contrast stretching, histogram equalization and CLAHE and can be beneficial for retinal vasculature segmentation. Contrast enhancement factor up to $\mathbf{2 . 6 2}$ for a digital retinal fundus image model is achieved. This improvement in contrast reduces the need of applying contrasting agent on patients.
Keywords: Contrast Enhancement; Independent Component Analysis; Medical Image Processing; Retinal Fundus Image

\section{INTRODUCTION}

Analyzing retinal fundus image is important for early detection of several diseases related to the retina, e.g. diabetic retinopathy. In diabetic retinopathy, retinal capillary occlusion occurs and accordingly causes enlargement of foveal avascular zone. Foveal avascular zone is the fovea where there is no blood vessels and located in the very centre of macula. Information of retinal vasculature is important to accurately determine the foveal avascular zone. However, digital color fundus images obtained from fundus camera suffer from several problems as can be seen from Figure 1. Figure 1(a) illustrates the problems of very low contrast and non-uniform illumination which can be seen at the area towards the edge of the image. Figure 1(b) shows the occurrence of noise which consists of impulse and Gaussian noises. Detection of the foveal avascular zone is even difficult due to very low image contrast of retinal vasculature against the background in the macular region.

A number of enhancement methods focused in the image spatial domain $[2,3,4,5]$. Histogram equalization with its modification is commonly used to enhance the image contrast [6]. However, histogram equalization tends to over-enhance the image and results in noisy appearance of the output image. One of the adaptive methods called contrast limited adaptive histogram equalization (CLAHE) worked well on the enhancement of retinal vasculature [7]. Iznita found that the contrast improvement using contrast limited adaptive histogram equalization on an image model ranges between 1.7 and 3 [8]. However, contrast limited adaptive histogram equalization creates artefacts in the enhanced image and the selection of contrast gain limit is image-dependent.

Other related works used the information of color taken from digital color images $[9,10]$. Colors observed 


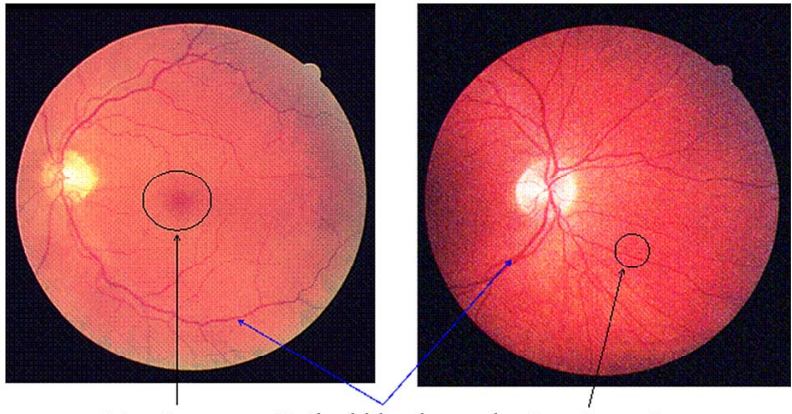

Macular area Retinal blood vessels Impulse noise

Figure 1. Digital fundus images obtained from fundus camera [1].

in the retinal image correspond to the architecture of retinal layer and the optical properties of the pigments $[11,12]$. Styles et al. developed a model using the concentrations of the five main absorbers found in the fundus layers, namely retinal haemoglobin, choroidal haemoglobin, choroidal melanin, retinal pigment epithetlium melanin and macular pigment [13]. This approach focuses more towards reconstructing rather than improving the contrast. Tsumura et al. showed that spatial distributions of melanin and haemoglobin from a skin color image can be separated using independent component analysis [9,14]. Nugroho et al. successfully applied a technique based on principal component analysis and independent component analysis to convert the RGB skin image into a skin image that represents skin areas due to melanin and haemoglobin only [10]. The above efforts focus on using independent component analysis to transform digital color image (RGB) into independent components that correspond to the biological makeup of the skin.

The objective of this work is to address the low contrast problem of retinal fundus images obtained from fundus camera when no contrasting agent is injected. A novel approach is presented to enhance the contrast of retinal vasculature by determining the retinal pigments, namely macular pigment, haemoglobin and melanin from fundus images. Distribution of haemoglobin is extracted from a fundus image to reveal retinal vasculature, which is a network of vessels in the retinal layer. Contrast of retinal vasculature obtained using this approach is compared to those from other enhancement methods, such as contrast stretching, histogram equalization and contrast limited adaptive histogram equalization to test the performance of this approach.

\section{APPROACH}

The approach taken in this research is as follows. First, a model of ocular fundus based on the light interaction is developed to describe the reflectance of the fundus. Second, a model of spectral absorbance of the retinal image is developed to show the components composing the observed colours in a digital fundus image. Third, inde- pendent component analysis based on the spectral absorbance of the model is applied to determine retinal pigments from fundus images. Finally, two fundus image models are developed to test performance of the proposed algorithm.

\subsection{Ocular Fundus Model}

Ocular fundus represents the structure of the back of the eyes that consists of multiple layers of tissue [13]. The ocular fundus image obtained from a fundus camera shows different intensity of reflectance. The reflectance depends on the wavelength, the structure of fundus' layers, the optical properties and quantities of retinal pigments in the ocular fundus. The incident light from a fundus camera can be reflected, absorbed, scattered or transmitted by the retinal tissues.

Generally, the structure of the eye can be classified into two main groups, namely ocular media and ocular fundus [15]. Ocular media consists of cornea, lens and vitreous. It is located between the ocular fundus and the observer. The ocular fundus consists of the retina, the retinal pigment epithelium, the choroid and the sclera. The reflectance of the fundus can be described in the terms of these layers [16]. Figure 2 depicts a model of ocular fundus showing possible pathways of the reflected light.

\subsection{Ocular Fundus Spectral Absorbance Model}

The spectral absorbance image provides useful information to identify the absorbance components [14]. In this work, we focus on the distribution of retinal pigments, namely haemoglobin, melanin, and macular pigment, rather than on the fundus layers, to model spectral absorbance of the ocular fundus [17].

Basis of linear combination of the absorption coefficients of melanin, haemoglobin and macular pigment is modelled from three absorbances $\mu_{\mathrm{a}}\left(\lambda_{1}\right), \mu_{\mathrm{a}}\left(\lambda_{2}\right)$ and

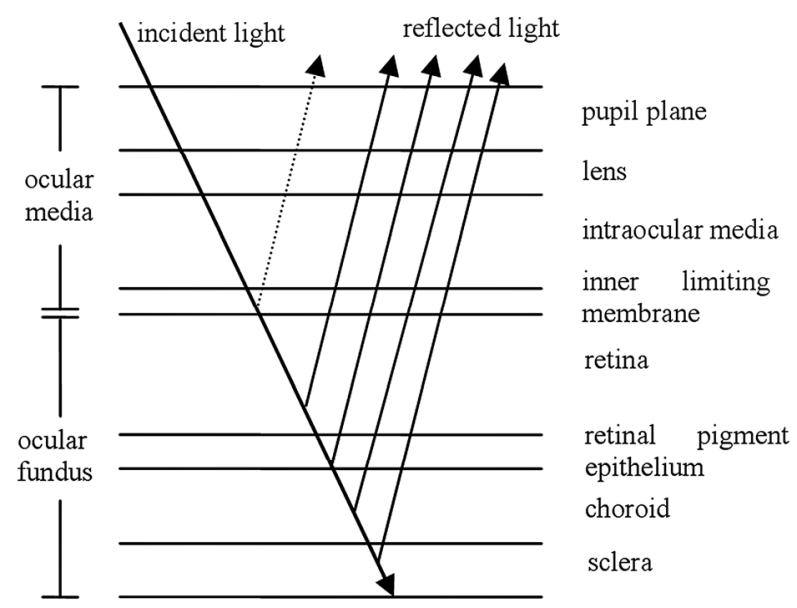

Figure 2. A model of ocular fundus showing pathways of reflected light. 
$\mu_{\mathrm{a}}\left(\lambda_{3}\right)$ at three wavelengths $\lambda_{1}, \lambda_{2}$ and $\lambda_{3}$. These wavelengths $\lambda_{1}, \lambda_{2}$ and $\lambda_{3}$ represent the red $(R)$, green $(G)$ and blue (B) color channels. Fundus spectral absorbance image shows spectral characteristics of the absorbance components in the ocular fundus. Two conditions are assumed when analyzing fundus spectral absorbances. First, the color observed in the fundus image is due to the distributions of melanin, haemoglobin and macular pigment. Second, the quantities of these components are spatially independent of each other. The spectral absorbance in the fundus image represents the linear combination of the absorption coefficients of melanin, haemoglobin and macular pigment.

Let $\boldsymbol{s}_{\mathrm{x}, \mathrm{y}}$ and $\boldsymbol{v}_{\mathrm{x}, \mathrm{y}}$ designate a three-dimensional (3-D) quantity vector and composite color vector on an image coordinate $(\mathrm{x}, \mathrm{y})$ of a digital color image. A mixing matrix $\mathbf{A}$ with $\boldsymbol{a}_{1}, \boldsymbol{a}_{2}$ and $\boldsymbol{a}_{3}$ represents pure color vectors of the three components (haemoglobin, melanin, macular pigment) per unit quantity. It is assumed that a linear combination of mutually independent pure color vectors with the quantities of $s_{1 \mathrm{x}, \mathrm{y}}, s_{2 \mathrm{x}, \mathrm{y}}$ and $s_{3 \mathrm{x}, \mathrm{y}}$ result in the composite color vectors of $\boldsymbol{v}_{1 \mathrm{x}, \mathrm{y}}, \boldsymbol{v}_{2 \mathrm{x}, \mathrm{y}}$ and $\boldsymbol{v}_{3 \mathrm{x}, \mathrm{y}}$ on the image coordinate $(\mathrm{x}, \mathrm{y})$. The following equation illustrates the transformation matrix, where $\mathrm{T}$ denotes the transpose.

$$
\begin{gathered}
\boldsymbol{v}_{x, y}=\mathbf{A} \boldsymbol{s}_{x, y} \\
\boldsymbol{s}_{x, y}=\left[s_{l x, y}, s_{2 x, y}, s_{3 x, y}\right]^{\mathrm{T}}
\end{gathered}
$$

The pixel value of each channel corresponds to each element of the color vector. Figure 3 depicts the spectral absorbances of the ocular fundus which consist of pure spectral vectors of melanin, haemoglobin and macular pigment.

\subsection{ICA of Fundus Spectral Absorbance Image}

Independent component analysis (ICA) is a technique to determine the original signals from mixtures of several independent sources $[18,19]$. The independent compo-

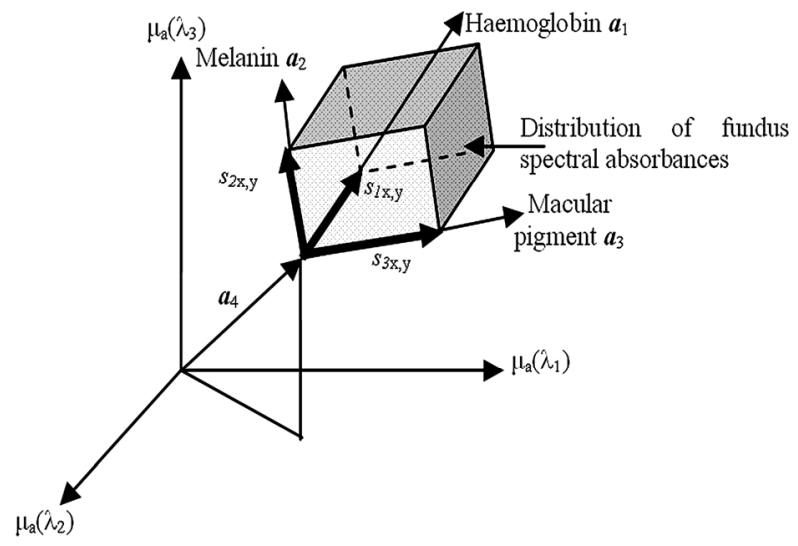

Figure 3. Model of spectral absorbance of the ocular fundus.

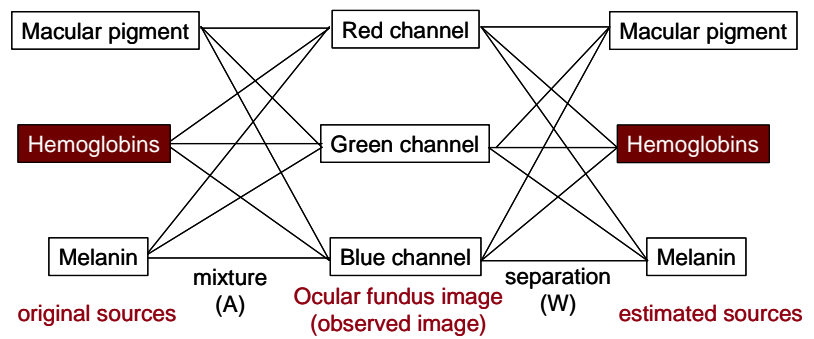

Figure 4. The problem of ICA in ocular fundus image.

nent analysis is modelled as

$$
\boldsymbol{v}=\mathbf{A s}
$$

with mixing matrix $\mathbf{A}$ and random vector $\boldsymbol{v}$, denoting the mixtures $v_{l}, v_{2}, \ldots, v_{n}$. Similarly, $\boldsymbol{s}$ random vector denotes the elements of $s_{1}, s_{2}, \ldots, s_{n}$. This model shows how the observed data $v_{n}$ is generated by a process of mixing the components $\boldsymbol{s}_{i}$. The independent components cannot be directly observed and neither can the mixing matrix. Only the random vector $\boldsymbol{v}$ is being observed. Mixing matrix $\mathbf{A}$ and random vector $\boldsymbol{s}$ are estimated using $\boldsymbol{v}$. Subsequently, separating matrix $\mathbf{W}$ is used to find the independent component simply by

$$
\hat{\boldsymbol{s}}=\mathbf{W} \boldsymbol{v},
$$

with $\hat{\boldsymbol{s}}$ is defined as estimated sources. The objective of independent component analysis is then to get $\hat{\boldsymbol{s}}$ as close as possible to $\boldsymbol{s}$, which is determined as original sources, by determining the optimum separating matrix $\mathbf{W}$. Mutually independent components are determined as elements of vector $s$ from the mixture of vectors in the image. A diagram is shown in Figure 4 to illustrate the idea of using independent component analysis in separating the spatial distributions of melanin, haemoglobin, and macular pigment in the ocular fundus. Three color channels, namely red, green and blue channels, represent random vector $\boldsymbol{v}$ and are used to determine these independent components [17]. By applying the independent component analysis to the composite colour vectors in the image, the relative quantity and pure colour vectors of each independent component are determined with no prior information on the quantity as well as colour vector. In this case, the independent components represent the retinal pigments, i.e. melanin, haemoglobin and macular pigment. The quantities of the retinal pigments are presumed to be mutually independent for the image coordinate. The separating matrix $\mathbf{W}$ is defined to separate vector $\hat{\boldsymbol{s}}_{\mathrm{x}, \mathrm{y}}$ using the following equations.

$$
\begin{gathered}
\hat{\mathbf{s}}_{\mathrm{x}, \mathrm{y}}=\mathbf{W}_{\mathbf{v}_{\mathrm{x}, \mathrm{y}}} \\
\hat{\mathbf{s}}_{\mathrm{x}, \mathrm{y}}=\left[\begin{array}{lll}
\hat{\mathrm{s}}_{1 \mathrm{x}, \mathrm{y}}, & \hat{\mathrm{s}}_{2 \mathrm{x}, \mathrm{y}}, & \hat{\mathrm{s}}_{3 \mathrm{x}, \mathrm{y}}
\end{array}\right]^{\mathrm{T}}
\end{gathered}
$$

The extracted independent components $\hat{s}_{1 \mathrm{x}, \mathrm{y}}, \hat{s}_{2 \mathrm{x}, \mathrm{y}}$ and $\hat{s}_{3 \mathrm{x}, \mathrm{y}}$ may be similar to $s_{I \mathrm{x}, \mathrm{y}}, s_{2 \mathrm{x}, \mathrm{y}}$ and $s_{3 \mathrm{x}, \mathrm{y}}$, respectively. The composite colour vector $\boldsymbol{v}_{\mathrm{x}, \mathrm{y}}$ is determined based on the logarithm transformation of the pixel intensities in 
the color channels of red, green and blue. Logarithmic transformation is used to transfer reflectance spectra into spectral absorbance since spectral absorbance image provides useful information to identify the absorbance components [14].

$$
\left[\mu_{\mathrm{a}}\left(\lambda_{1}\right), \mu_{\mathrm{a}}\left(\lambda_{2}\right), \mu_{\mathrm{a}}\left(\lambda_{3}\right)\right]=\left[-\log \left(r_{\mathrm{x}, \mathrm{y}}\right),-\log \left(g_{\mathrm{x}, \mathrm{y}}\right),-\log \left(b_{\mathrm{x}, \mathrm{y}}\right)\right]
$$

here, the values of $r_{\mathrm{x}, \mathrm{y}}, g_{\mathrm{x}, \mathrm{y}}$ and $b_{\mathrm{x}, \mathrm{y}}$ correspond to pixel intensity in the color channels of red, green and blue respectively. The composite color vector is denoted as

$$
v_{\mathrm{x}, \mathrm{y}}=\left[\mu_{\mathrm{a}}\left(\lambda_{1}\right), \mu_{\mathrm{a}}\left(\lambda_{2}\right), \mu_{\mathrm{a}}\left(\lambda_{3}\right)\right]^{\mathrm{T}}
$$

According to the model of spectral absorbance in the ocular fundus from Figure 3, the color density vector of the fundus can be stated as

$$
\boldsymbol{v}_{\mathrm{x}, \mathrm{y}}=\mathbf{A} \boldsymbol{s}_{\mathrm{x}, \mathrm{y}}+\boldsymbol{a}_{4}
$$

where $\mathbf{A}=\left[\boldsymbol{a}_{1}, \boldsymbol{a}_{2}, \boldsymbol{a}_{3}\right]$ and $\boldsymbol{s}_{x, y}=\left[s_{1 \mathrm{x}, \mathrm{y}}, s_{2 \mathrm{x}, \mathrm{y}}, s_{3 \mathrm{x}, \mathrm{y}}\right]^{\mathrm{T}}$. Elements $\boldsymbol{a}_{1}, \boldsymbol{a}_{2}$ and $\boldsymbol{a}_{3}$ of the mixing matrix $\mathbf{A}$ represents pure color vectors of the three components (haemoglobin, melanin, macular pigment) per unit quantity. It is assumed that a linear combination of mutually independent pure color vectors with the quantities of $s_{1 \mathrm{x}, \mathrm{y}}, s_{2 \mathrm{x}, \mathrm{y}}$ and $s_{3 \mathrm{x}, \mathrm{y}}$ results in the composite color vectors of $\boldsymbol{v}_{1 \mathrm{x}, \mathrm{y}}$, $\boldsymbol{v}_{2 \mathrm{x}, \mathrm{y}}$ and $\boldsymbol{v}_{3 \mathrm{x}, \mathrm{y}}$ on the image coordinate $(\mathrm{x}, \mathrm{y})$. Additionally, $\boldsymbol{a}_{4}$ is similar to noise in the ICA model. In this case, the model is assumed to be noise-free, therefore $\boldsymbol{a}_{4}$ can be neglected.

Several methods, such as fast fixed-point algorithm (FastICA) [20], joint approximate diagonalization of eigen-matrices (JADE) [21] and information- maximization (infomax) [22] have been proposed to solve the problem of independent component analysis. In ICA, the only assumption needed are: 1) the sources are statistically independent, 2) the probability densities of the sources are non-Gaussian, 3) the mixing of the sources into the observations is linear, and 4) the number of observations is larger than or equal to he number of sources [19]. The FastICA algorithm with symmetrical orthogonalization is used to get the estimated independent components because of its good accuracy and high computational speed for high dimensional data [20].

\subsection{Fundus Image Model}

A model of fundus image is developed to test the performance of independent component analysis in separating the distribution of macular pigment, hemoglobin and melanin. Mixture of three mutually independent components, i.e. macular pigment, hemoglobin and melanin is used to model a fundus image. As shown in Table 1, the statistical intensity description of macular pigment, haemoglobin and melanin in red, green and blue channels are taken from the 44 test images from FINDeRS [23]. A smaller region containing macular area is sampled to get the probability density function of the
Table 1. Statistical intensity description of macular pigment, haemoglobin and melanin in red $(\mathrm{R})$, green $(\mathrm{G})$ and blue $(\mathrm{B})$ channels.

\begin{tabular}{cccc}
\hline & $\begin{array}{c}\text { Macular } \\
\text { pigment }\end{array}$ & Haemoglobin & Melanin \\
\hline Mean R & 97.14868 & 120.0417 & 156.5642 \\
$\begin{array}{c}\text { Standard } \\
\text { deviation R }\end{array}$ & 28.39299 & 27.16076 & 23.98799 \\
Skewness R & 0.714194 & 0.74299 & 0.07827 \\
Kurtosis R & 0.522469 & 0.259348 & 0.215941 \\
Minimum R & 46.26375 & 73.32877 & 102.7158 \\
Maximum R & 174.1571 & 195.1525 & 219.6025 \\
Mean G & 48.29849 & 62.24773 & 96.08492 \\
Standard & 13.82747 & 17.29835 & 19.95184 \\
deviation G & 0.719616 & 0.376683 & 0.620011 \\
Skewness G & 1.577469 & 0.236853 & 1.59036 \\
Kurtosis G & 21.67789 & 32.06349 & 57.14166 \\
Minimum G & 95.15525 & 114.2881 & 164.2989 \\
Maximum G & 7.971792 & 17.48195 & 35.1042 \\
Mean B & 5.483867 & 11.73761 & 18.85215 \\
Standard \\
deviation B & 1.989686 & 1.596864 & 1.31945 \\
Skewness B & 6.239178 & 3.978853 & 2.216477 \\
Kurtosis B & 1.992481 & 2.412698 & 12.66622 \\
Minimum B & 31.36347 & 63.40678 & 100.4873 \\
\hline Maximum B & & &
\end{tabular}

retinal pigments. In the macular region, retinal capillaries usually show a very low contrast between retinal blood vessels and the background. A clustering method using k-means based on the intensity of red, green, and blue channels of the macular pigment, haemoglobin and melanin is performed to classify the samples due to large value of standard deviation and intensity range of the sample. Based on the experiment, two numbers of clusters are found to be optimal to classify the samples.

In Figure 5, two fundus image models to represent fair and dark fundus images with mixture of specified sample intensity distribution of macular pigment, haemoglobin and melanin in red, green and blue channels are shown. Using these models as the input, the independent component analysis should be able to separate these components into three outputs, namely macular pigment, haemoglobin and melanin.

\section{RESULTS AND DISCUSSIONS}

A fundus image model is firstly tested using independent component analysis to see performance of the algorithm. The inputs to the FastICA are three separate channels (i.e. red, green and blue channels) of a color fundus image model. As can be seen from Figure 6, the proposed algorithm successfully separates the compo- 


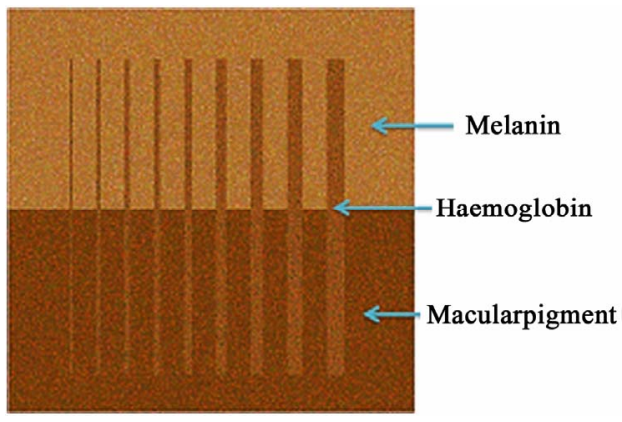

(a). Fair image model

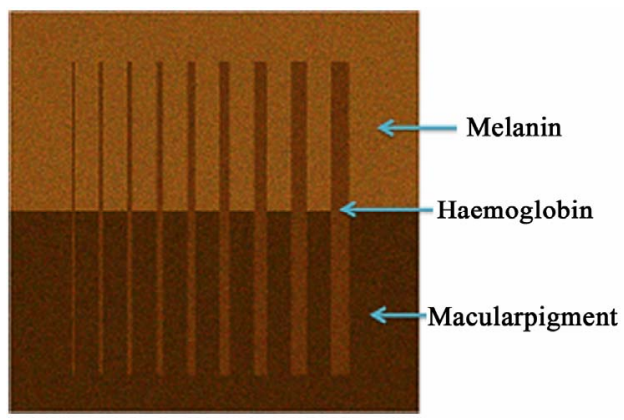

(b). Dark image model

Figure 5. Fundus image model.

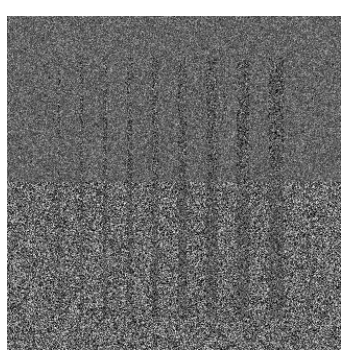

a. Macular region

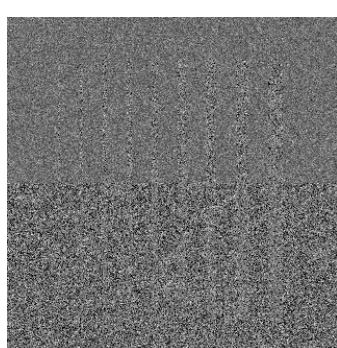

b. Melanin

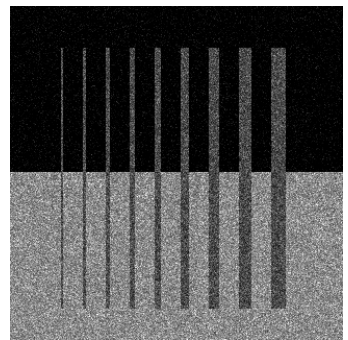

c. Retinal vasculature

Figure 6. Independent component analysis of dark fundus image model.

nents into three, namely macular pigment, haemoglobin and melanin. These three independent components represent macular region, retinal vasculature and melanin, respectively. In Figure 6(a), the brighter area in lower part of the fundus image model is related to the macular region. In Figure $\mathbf{6}(\mathbf{b})$, the melanin is illustrated as the brighter area in upper part of the fundus image model. These two components can be clearly distinguished from the other component, which is related to retinal vasculature, since the retinal vasculature is almost invisible in the appearance of these two components (i.e. macular region and melanin). Furthermore, the retinal vasculature is clearly visualized in Figure 6(c). As a result, independent component image due to haemoglobin obtained exhibits higher contrast retinal vasculature compared to that of the original image.

In this work, 44 retinal fundus images containing macular region are taken from FINDeRS database to model a retinal fundus image. The fundus image model undergoes several enhancement methods, such as contrast stretching, histogram equalization, contrast limited adaptive histogram equalization (CLAHE) to measure contrast improvement factor of these methods and compare to the proposed algorithm. A smaller region containing the macular area is taken to see the enhancement of retinal capillaries, which usually show a very low contrast between retinal blood vessels and the background. Figure 7 shows green band of dark fundus image model undergoing several enhancement methods, i.e. contrast stretching, histogram equalization and CLAHE. Qualitatively, haemoglobin related ICA shows better enhancement because no artefacts is produced in the process. Nevertheless, the other three enhancement methods tend to increase the noise presence in the image as well as to produce artefacts.

From the fundus image model, the green band image shows the average contrast intensity of 24.60 and 16.80 for fair and dark image model, respectively. Using these values as a reference, the proposed algorithm using ICA

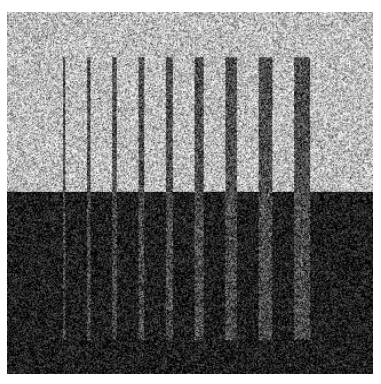

a. Contrast stretching

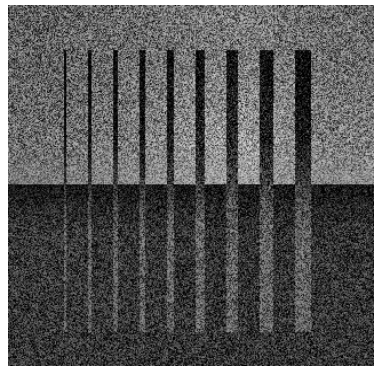

c. Contrast limited AHE

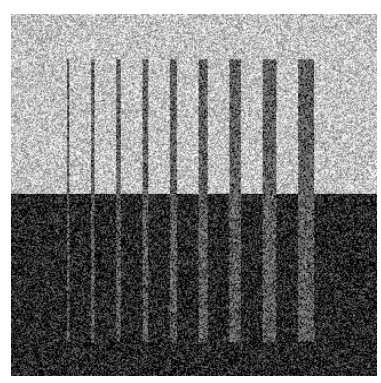

b. Histogram equalization

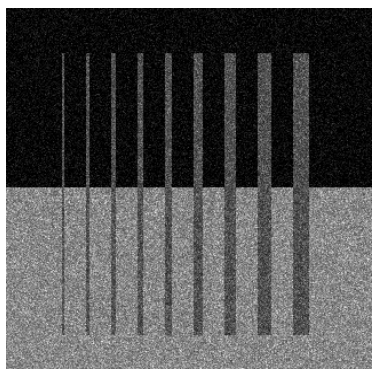

d. ICA (haemoglobin)
Figure 7. Dark fundus image model undergoes several enhancement methods.

with contrast enhancement factor of 1.47 and 2.62 for 
fair and dark fundus image models shows a better improvement for both fair and dark fundus image models than that of the other three enhancement methods. Furthermore, as is shown in Figure 8, CLAHE with enhancement factor of 1.37 and 1.98 for fair and dark fundus image model, respectively, is still better than that of contrast stretching and histogram equalization. However, compared to that of CLAHE, the proposed algorithm produces no artefacts in the process.

Here an example of retinal image showing macular region is taken to see enhancement of retinal vasculature using the proposed algorithm. In a preliminary work using the above algorithm, it is found that non-uniform illumination in fundus images resulted in false detection of the retinal pigments [17]. This is because the algorithm responds to the spectral reflectance or absorbance of the retinal pigments in the image. Therefore, homomorphic filtering is performed prior to independent component analysis to reduce the problem of non-uniform illumination. Homomorphic filtering is used to reduce illumination which varies slowly in space and at the same time [24].

Figure 9 shows an original color fundus image undergoing homomorphic filtering and its independent components estimated by the FastICA algorithm. The components represent the distribution of the pigments, namely macular pigment, haemoglobin and melanin. The brighter area in the centre of the first independent component (Figure 9(b)) represents the distribution of macular pigment. The second independent component (Figure 9(c)) shows the distribution of haemoglobin. It is indicated by the enhancement of retinal vasculature. The third independent component (Figure 9(d)) shows brighter area related to the distribution of melanin. This result is consistent with the location of melanin, which is fairly distributed in the retinal pigment epithelium and the choroid. Based on the assumption that the image is noise-free, independent component analysis is able to determine the retinal pigments. Moreover, as shown in Figure 10, a green band image undergoing CLAHE is

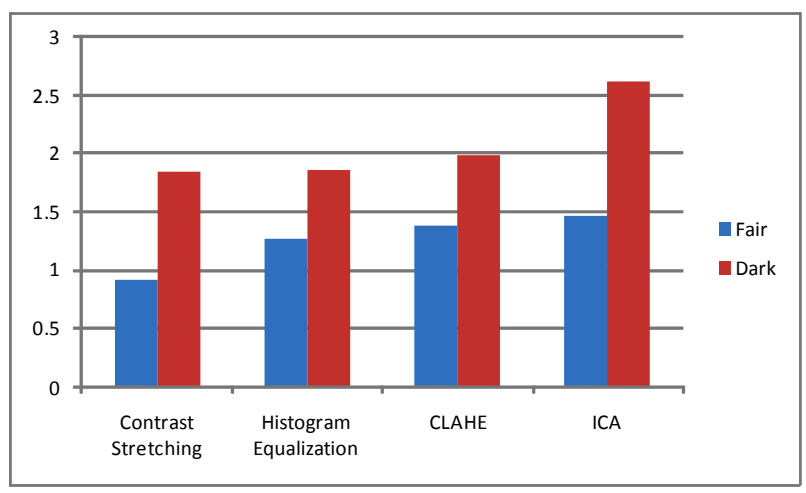

Figure 8. Contrast enhancement factor of retinal vasculature in fundus image model.

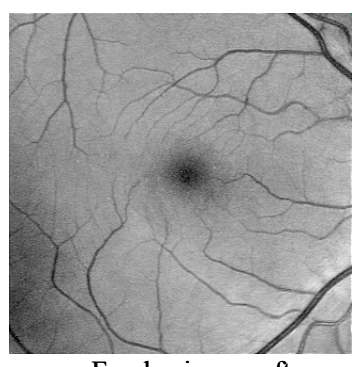

a. Fundus image after homomorphic filtering

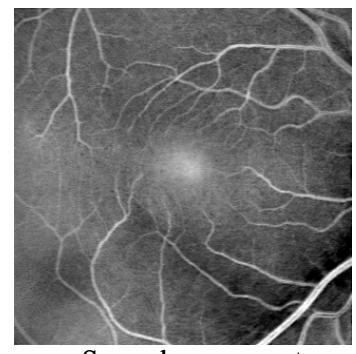

c. Second component

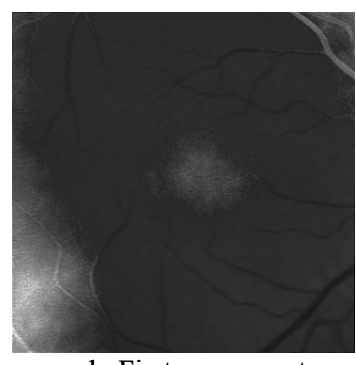

b. First component

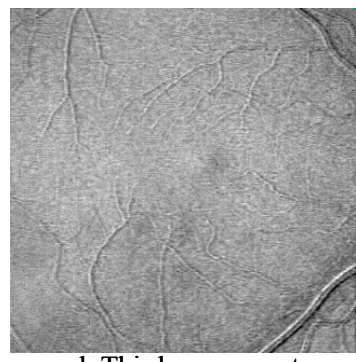

d. Third component
Figure 9. Independent component analysis of a retinal image containing macular region.

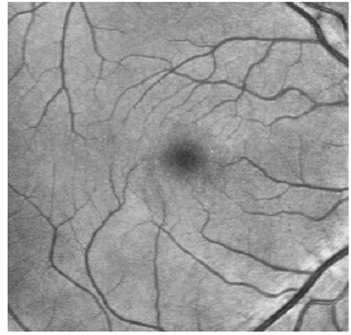

a. Green band image after CLAHE

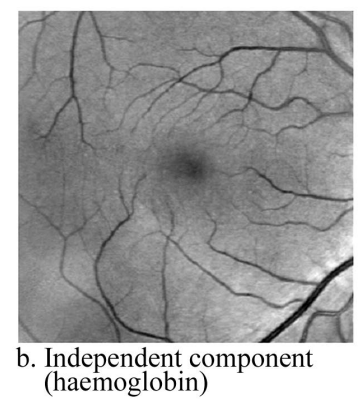

(haemoglobin)
Figure 10. Comparison of contrast enhancement of retinal vasculature between CLAHE and ICA.

compared to the haemoglobin-related component image after the intensity is being inverted to demonstrate that contrast enhancement is also achieved. In this work, CLAHE is also performed on the same images undergoing the proposed algorithm to compare the contrast improvement between these two methods. Having measured the contrast improvement factor on the fundus image model, the proposed algorithm consistently shows better visualization and enhancement compared to that of the CLAHE, which is commonly used as pre-processing for segmentation of retinal vasculature in fundus images. This improvement can be beneficial to improve the accuracy of retinal vasculature segmentation and reduce the need for injecting contrasting agent to the patients.

\section{CONCLUSIONS}

Analyzing retinal fundus images is usually difficult as they are of very low contrast. Low contrast between blood vessels and the background makes it difficult to 
accurately determine retinal vasculature. Retinal vasculature can be used to determine existence of pathology, macular area and foveal avascular zone. Typical contrast enhancement methods usually create artefacts or introduce noise. Even though fluorescein angiography produces better contrast enhancement, it is not preferable due to its invasive nature of injecting contrasting agent.

In this work, the developed method based on the spectral absorbance model and independent component analysis enables us to determine the retinal pigments, namely haemoglobin, melanin and macular pigment. A fundus image model has been developed to test the performance of the proposed algorithm. As a result, retinal vasculature, macular pigment and melanin distribution can be determined from digital fundus image. Results show that this approach outperforms other non-invasive enhancement methods, such as contrast stretching, histogram equalization and CLAHE and can be beneficial for vessel segmentation. The algorithm produces no artefacts in the process. Using the haemoglobin component, the contrast between retinal blood vessels and the background can be enhanced with contrast enhancement factor up to 2.62 for a model of fundus image. This improvement in contrast reduces the need of applying contrasting agent on patients.

\section{REFERENCES}

[1] Staal, J., Abramoff, M. D., Niemeijer, M., Viergever, M. A., and Ginneken van B., (2004) Ridge-based vessel segmentation in color images of the retina, Medical Imaging, IEEE Transactions, 23, 501-509.

[2] Niemeijer, M., Staal, J., Ginneken v. B., Loog M., and Abramoff M. D., (2004) Comparative study of retinal vessel segmentation methods on a new publicly available database, Medical Imaging: Image Processing, Milan, 648-656.

[3] Shimahara, T., Okatani, T., and Deguchi, K., (2004) Contrast enhancement of fundus images using regional histograms for medical diagnosis, SICE Annual Conference, 1, 650-653.

[4] Sinthanayothin, C., Kongbunkiat, V., Phoojaruenchanachai, S., and Singalavanija, A., (2003) Automated screening system for diabetic retinopathy, Image and Signal Processing and Analysis, ISPA, Proceedings of the 3rd International Symposium on, 915.

[5] Wu, D., Zhang, M., Liu, J. C., and Bauman, W., (2006) On the adaptive detection of blood vessels in retinal images, Biomedical Engineering, IEEE Transactions on, 53, 341-343.

[6] Yang, S., Oh, J. H., and Park, Y., (2003) Contrast enhancement using histogram equalization with bin underflow and bin overflow, in Image Processing, ICIP, Proceedings International Conference on, 1, I-881-4.

[7] Fadzil, M. H. A., Izhar, L. I., Venkatachalam, P. A., and Karunakar, T. V. N., (2007) Extraction and reconstruction of retinal vasculature, Journal of Medical Engineering Technology, 31, 435-442.

[8] Iznita, L., (2006) Analysis of retinal vasculature and foveal avascular zone for grading of diabetic retinopathy, in M.Sc, Thesis Electrical and Electronics Engineering Programme Bandar Seri Iskandar, Malaysia, Universiti Teknologi PETRONAS.

[9] Tsumura, N., Haneishi, H., and Miyake, Y., (1999) Independent-component analysis of skin color image, J. Opt. Soc. Am. A, 16, 2169.

[10] Nugroho, H., Fadzil, M. H. A., Yap, V. V., Norashikin, S., and Suraiya, H. H., (2007) Determination of Skin Repigmentation Progression, Engineering in Medicine and Biology Society, EMBS Annual International Conference of the IEEE, 3442.

[11] Preece, S. J. and Claridge, E., (2002) Physics Based Medical Image Understanding of the Colouration of the Ocular Fundus with Application to Detection of Diabetic Retinopathy, Department of Computer Science, University of Birmingham, Edgbaston, Birmingham Technical Report CSR-04-08.

[12] Orihuela-Espina, F., Claridge, E., and Preece, S. J., (2003) Histological parametric maps of the human ocular fundus: preliminary results, Medical Image Understanding and Analysis, 133-136.

[13] Styles, I. B., Calcagni, A., Claridge, E., Orihuela-Espina, F., and Gibson, J. M., (2006) Quantitative analysis of multi-spectral fundus images, Medical Image Analysis: Special Issue on Functional Imaging and Modelling of the Heart (FIMH 2005), 10, 578-597.

[14] Tsumura, N., Haneishi, H., and Miyake, Y., (2000) Independent Component Analysis of Spectral Absorbance Image in Human Skin, Optical Review, 7, 479-482.

[15] Knighton, R. W., (1995) Quantitative reflectometry of the ocular fundus, Engineering in Medicine and Biology Magazine, IEEE, 14, 43-51.

[16] Berendschot, T. T. J. M., DeLint, P. J., and Norren, D., (2003) Fundus reflectance-historical and present ideas, Progress in Retinal and Eye Research, 22, 171-200.

[17] Ahmad Fadzil, M. H., Nugroho, H. A., Venkatachalam, P. A., Nugroho, H., and Izhar, L. I., (2008) Determination of Retinal Pigments from Fundus Images using Independent Component Analysis, in Biomed 4th Kuala Lumpur International Conference on Biomedical Engineering, Kuala Lumpur, 555-558.

[18] Comon, P., (1994) Independent component analysis, A new concept?, Signal Processing, 36, 287.

[19] Hyvarinen, A. and Oja, E., (2000) Independent component analysis: algorithms and applications, Neural Networks, 13, 411.

[20] Hyvarinen, E., (1999) Fast and robust fixed-point algorithms for independent component analysis, Neural Networks, IEEE Transactions on, 10, 626-634.

[21] Cardoso, J. F. and Souloumiac, A., (1999) Blind beamforming for non Gaussian signals, IEE Proceedings-F, 140, 362-370.

[22] Bell, A. J. and Sejnowski, T. J., (1995) An information-maximisation approach to blind separation and blind deconvolution, Neural Computation, 7, 1129-1159.

[23] Ahmad Fadzil, M. H., Nugroho, H. A., Nugroho, H., and Izhar, L. I., (2009) Fundus Image Database for Non Invasive Diabetic Retinopathy Monitoring and Grading System (FINDeRS), Universiti Teknologi PETRONAS.

[24] Adelmann, H. G., (1998) Butterworth equations for homomorphic filtering of images, Computers in Biology and Medicine, 28, 169-181. 